\title{
A novel automated strategy for screening cryptic telomeric rearrangements in children with idiopathic mental retardation
}

\author{
Laurence Colleaux*,1, Marlène Rio ${ }^{1}$, Solange Heuertz ${ }^{1}$, Séverine Moindrault ${ }^{4}$, \\ Catherine Turleau ${ }^{1}$, Catherine Ozilou ${ }^{3}$, Philippe Gosset ${ }^{3}$, Odile Raoult ${ }^{3}$, Stanislas Lyonnet $^{2}$, \\ Valérie Cormier-Daire ${ }^{2}$, Jeanne Amiel ${ }^{2}$, Martine Le Merrer ${ }^{2}$, Monique Picq ${ }^{3}$, \\ Marie-Christine de Blois ${ }^{3}$, Marguerite Prieur ${ }^{3}$, Serge Romana ${ }^{3}$, François Cornelis ${ }^{4}$, \\ Michel Vekemans ${ }^{3}$ and Arnold Munnich ${ }^{1}$
}

${ }^{1}$ INSERM U393, Hôpital Necker-Enfants Malades, Paris, France; ${ }^{2}$ Service de Génétique Médicale, Hôpital NeckerEnfants Malades, Paris, France; ${ }^{3}$ Département de Cytogénétique, Hôpital Necker-Enfants Malades, Paris, France; ${ }^{4}$ ECRAF-Université Paris 7, GENOPOLE, Evry, France

Cryptic unbalanced subtelomeric rearrangements are known to cause a significant proportion of idiopathic mental retardation in childhood. Because of the limited sensitivity of routine analyses, the cytogenetic detection of such rearrangements requires molecular techniques, namely FISH and comparative genomic hybridisation $(\mathrm{CGH})$. An alternative approach consists in using genetic markers to detect segmental aneusomy. Here, we describe a new strategy based upon automated fluorescent genotyping to search for non mendelian segregation of telomeric microsatellites. A total of 29 individuals belonging to 24 unrelated families were screened and three abnormal patterns of segregation were detected (two rearrangements and one parental disomy). This study gives strong support to the view that cryptic telomeric rearrangements significantly contribute to idiopathic mental retardation and demonstrates that fluorescent genotyping is a very sensitive and cost-effective method to detect deletions, duplications and uniparental disomies. European Journal of Human Genetics (2001) 9, 319-327.

Keywords: mental retardation; telomere; chromosome rearrangement; automated genotyping; uniparental disomy

\section{Introduction}

Despite recent advances, the origin of largely $40 \%$ of moderate to severe cases of mental retardation (defined by an IQ score <50) remains unexplained. ${ }^{1,2}$ Recognizing the cause of mental retardation has little therapeutic impact but helps in clarifying the recurrence risk and makes prenatal diagnosis and carrier testing feasible in affected families. Segmental aneusomy has recently been recognised as a significant cause of mental retardation. ${ }^{3}$ However, standard

*Correspondence: Dr Laurence Colleaux, INSERM U393, Tour Lavoisier, Hôpital Necker-Enfants Malades, 149 rue de Sèvres, 75015 Paris, France. Tel: +33 1444951 63; Fax: +33 1444951 50; E-mail: colleaux@necker.fr Received 29 May 2000; revised 24 October 2000; accepted 26 October 2000 cytogenetic analyses have only a 400-500 band resolution and may overlook subtle chromosome rearrangements in individuals with apparently normal karyotype. Indeed, cryptic subtelomeric rearrangements have eventually been observed in the alpha-thalassemia/mental retardation syndrome, $^{4,5}$ the Wolf-Hirschhorn syndrome (MIM 194190, deletion of terminal 4p), ${ }^{6}$ the Miller-Dieker syndrome (MIM 247200 , deletion of terminal $17 \mathrm{p}){ }^{7}$ or the cri-du-chat syndrome (MIM-123450, deletion of terminal $5 \mathrm{p}) .{ }^{8}$ Higher resolution analyses (850-band resolution) are feasible but they are time-consuming and rather more suited for the analysis of specific chromosomal regions.

For this reason, a molecular cytogenetic approach based on fluorescence in-situ hybridization with telomere-specific probes and a multiprobe coverslip has been developed, ${ }^{9}$ and 
has led to the conclusion that subtle chromosomal abnormalities occur in $7.4 \%$ of severely retarded patients. ${ }^{10}$ However, this method requires the preparation of high mitotic index chromosomes and remains difficult and expensive for routine diagnostic tests. Similarly, comparative genomic hybridisation (CGH) is a valuable technique but this approach is unable to detect subtle anomalies (below $5 \mathrm{Mb}$ ) and requires specific microscope and computer facilities. ${ }^{11}$

On the other hand, microsatellite markers are naturally occurring DNA polymorphisms that can be used to search for irregular allele inheritance and detection of deletions, duplications and uniparental disomies. ${ }^{12}$ Recently, a pilot study based on conventional genotyping allowed diagnosis of two deletions of telomeric regions in a series of 27 children with idiopathic mental retardation. ${ }^{13}$ We have developed a novel strategy based on automated fluorescent genotyping using a new set of telomere-specific markers. Here, we show that automated fluorescent genotyping can be successfully adapted to the detection of cryptic terminal unbalanced translocations and uniparental disomies in patients with idiopathic mental retardation. This approach will hopefully help to determine the actual frequency of these rearrangements in idiopathic mental retardation and may lead to the identification of unknown disease genes and imprinted regions.

\section{Materials and methods \\ Patients}

A total of 29 children ( 17 boys and 12 girls, belonging to 24 families) born to unrelated parents and presenting with moderate or severe idiopathic mental retardation (IQ below 50) and non-syndromic dysmorphic features were recruited from the Department of Genetics of the Hopital Necker. Inclusion criteria were the presence of at least one of the following: (1) seizures; (2) overgrowth or failure to thrive; (3) behavioural anomalies (hyperactivity, aggressiveness or automutilation; and (4) congenital anomalies. In addition, all patients had a normal karyotype using both RHG and GTG banding analysis at 500-550 band resolution level. Their clinical features are summarised in Table 1.

\section{Microsatellite markers, PCR amplification and GeneScan analysis}

The number, location and heterozygosity scores of the microsatellites are shown in Table 2 and the data were obtained from either the GENETHON database (http:// www.genethon.fr/) or the GENOME DATABASE (http:// www.gdb.org/). Primers labelled with a NED fluorochrome were ordered from PE Biosystem (Courtabeuf, France) and primers labelled with a 6-FAM or HEX fluorochrome were ordered from MWG BIOTECH (Courtabeuf, France). DNA was extracted from peripheral blood lymphocytes according to standard techniques. PCR amplification of genomic DNA from parents and children (100 ng) was separately performed in 96-well microtitre plates using a Perkin-Elmer 9700 thermocycler and the PE Biosystems AmpliTaq Gold ${ }^{\mathrm{TM}}$ according to manufacturer's instructions. PCR products were then pooled according to their size and labelling (see Results) and analysed on an automatic sequencer (ABI 377, PE Biosystem) according to the manufacturer's recommenda-

Table 1 Summary of rearrangements and associated phenotype

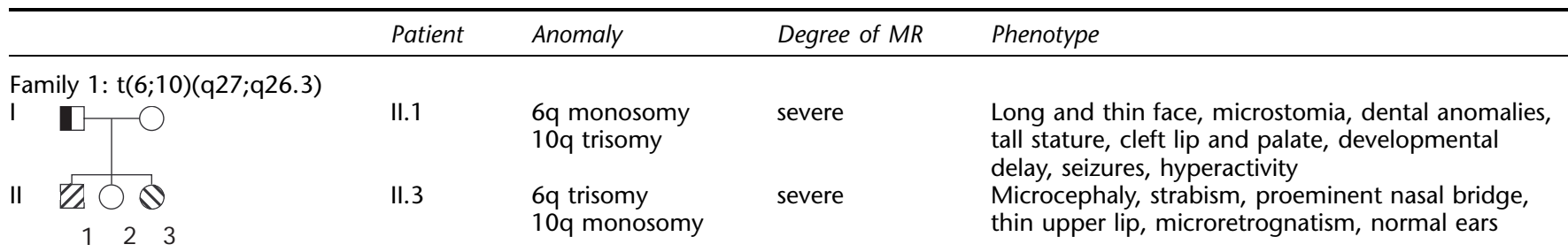

Family 3: del 10q26.3

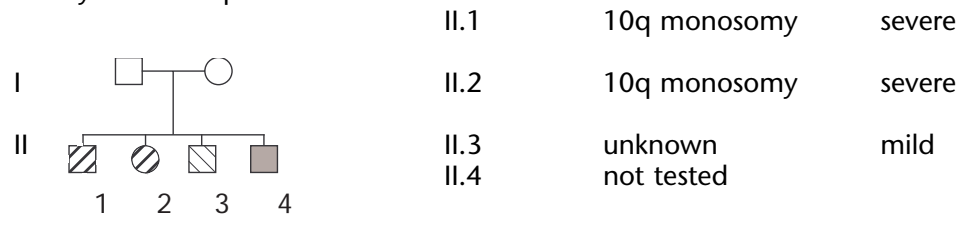

Enophtalmia, long nose, full lips and everted lower lip, foot deformation, autism Enophtalmia, long nose, full lips and everted lower lip, foot deformation, autism No dysmorphy, normal language Died at age 2 years

Family 4: UPD of $17 q 25.3$

I

Macrostomia, thick upper lip, hypertelorism epicanthus, broad nasal bridge, aggressive behaviour

$\square$, unaffected carrier of a balanced translocation; $\mathbb{Z}, \mathbb{Q}$, two different unbalanced chromosomal rearrangements derived from the balanced translocation; 
Table 2 Microsatellite markers used for telomere genotyping

\begin{tabular}{|c|c|c|c|c|c|}
\hline Telomere & $\begin{array}{l}\text { D } \\
\text { Number }\end{array}$ & $\begin{array}{l}\text { Genetic } \\
\text { distance } \\
\text { to } \\
\text { telomere } \\
(\mathrm{cM})\end{array}$ & Fluorophore & Size range & $\begin{array}{l}\text { Hetero- } \\
\text { zygosity }\end{array}$ \\
\hline \multirow[t]{2}{*}{$1 p$} & D1S243 & 0 & FAM & $142-170$ & 0.86 \\
\hline & D1S468 & 6.2 & HEX & $173-191$ & 0.75 \\
\hline \multirow[t]{2}{*}{$1 q$} & D1S2682 & 0 & NED & $110-150$ & 0.76 \\
\hline & D1S2836 & 2.6 & FAM & $268-281$ & 0.79 \\
\hline \multirow[t]{2}{*}{$2 p$} & D2S2268 & 0 & HEX & $205-231$ & 0.61 \\
\hline & D2S323 & 3.6 & FAM & $177-193$ & 0.57 \\
\hline \multirow[t]{2}{*}{$2 q$} & D2S2338 & 0 & FAM & $155-173$ & 0.55 \\
\hline & D2S140 & 4.5 & NED & $151-167$ & 0.76 \\
\hline \multirow[t]{2}{*}{$3 p$} & D3S1270 & 0 & NED & $154-196$ & 0.75 \\
\hline & D3S1307 & 1.4 & FAM & $237-251$ & 0.80 \\
\hline \multirow[t]{2}{*}{$3 q$} & D3S3550 & 0 & FAM & $230-270$ & 0.74 \\
\hline & D3S3707 & 2.2 & HEX & $262-278$ & 0.65 \\
\hline \multirow[t]{2}{*}{$4 p$} & D4S3038 & 0 & NED & $195-240$ & 0.78 \\
\hline & D4S2936 & 0 & NED & $170-184$ & 0.83 \\
\hline \multirow[t]{2}{*}{$4 q$} & D4S2930 & 0 & NED & $216-236$ & 0.80 \\
\hline & D4S426 & 1.1 & HEX & $177-191$ & 0.56 \\
\hline \multirow[t]{2}{*}{$5 p$} & D5S678 & 0 & FAM & $252-264$ & 0.71 \\
\hline & D5S392 & 0.6 & HEX & $83-117$ & 0.87 \\
\hline \multirow[t]{2}{*}{$5 q$} & D5S2006 & 0 & HEX & $135-165$ & 0.74 \\
\hline & D5S408 & 1.8 & FAM & $247-265$ & 0.73 \\
\hline \multirow[t]{2}{*}{$6 p$} & D6S1600 & 0 & FAM & $165-210$ & 0.77 \\
\hline & D6S344 & 1.4 & HEX & $139-159$ & 0.72 \\
\hline \multirow[t]{2}{*}{$6 q$} & D6S281 & 0 & HEX & $125-180$ & 0.68 \\
\hline & D6S1693 & 10.8 & FAM & $85-93$ & 0.51 \\
\hline \multirow[t]{2}{*}{$7 p$} & D7S2477 & 0 & FAM & $132-180$ & 0.66 \\
\hline & D7S2563 & 1.7 & HEX & $91-111$ & 0.78 \\
\hline \multirow[t]{2}{*}{$7 q$} & D7S2423 & 0 & NED & $230-270$ & 0.71 \\
\hline & D7S2465 & 1.9 & HEX & $158-181$ & 0.83 \\
\hline \multirow[t]{2}{*}{$8 p$} & D8S504 & 0 & FAM & $193-203$ & 0.71 \\
\hline & D8S264 & 0.7 & FAM & $121-145$ & 0.83 \\
\hline \multirow[t]{2}{*}{$8 q$} & D8S1836 & 0 & HEX & $121-160$ & 0.84 \\
\hline & D8S1751 & 1.4 & NED & $149-163$ & 0.69 \\
\hline $9 p$ & D9S1779 & 0 & NED & $114-154$ & 0.63 \\
\hline & D9S1858 & 0.1 & HEX & $124-144$ & 0.58 \\
\hline $9 q$ & D9S1838 & 0 & NED & $155-175$ & 0.83 \\
\hline & D9S158 & 3.5 & FAM & $213-233$ & 0.71 \\
\hline $10 p$ & D10S249 & 0 & NED & $106-152$ & 0.74 \\
\hline & D10S558 & 1.9 & NED & $192-212$ & 0.80 \\
\hline $10 q$ & D10S1700 & 0 & HEX & $107-148$ & 0.64 \\
\hline & D10S212 & 1 & HEX & $189-201$ & 0.70 \\
\hline $11 p$ & D11S1363 & 0 & FAM & $240-260$ & 0.59 \\
\hline & D11S4177 & 3.1 & FAM & $183-213$ & 0.80 \\
\hline $11 q$ & D11S968 & 3.3 & HEX & $131-165$ & 0.81 \\
\hline & D11S4125 & 3.3 & FAM & $231-253$ & 0.81 \\
\hline $12 p$ & D12S352 & 0 & HEX & $145-170$ & 0.72 \\
\hline & D12S341 & 0.6 & FAM & $114-130$ & 0.79 \\
\hline $12 q$ & D12S357 & 0 & HEX & $260-315$ & 0.84 \\
\hline & D12S1638 & 0 & NED & $120-130$ & 0.68 \\
\hline $13 q$ & D13S293 & 0 & NED & $80-106$ & 0.50 \\
\hline & D13S1295 & 4.6 & HEX & $102-120$ & 0.74 \\
\hline $14 q$ & D14S1007 & 0 & HEX & $110-135$ & 0.77 \\
\hline & D14S293 & 3.9 & HEX & $153-165$ & 0.65 \\
\hline $15 q$ & D15S966 & 0 & HEX & $225-265$ & 0.82 \\
\hline & D15S203 & 0.6 & FAM & $116-140$ & 0.62 \\
\hline $16 p$ & D16S521 & 0 & FAM & $156-172$ & 0.71 \\
\hline & D16S3024 & 5 & HEX & $208-248$ & 0.86 \\
\hline $16 q$ & D16S3121 & 0 & HEX & $65-90$ & 0.62 \\
\hline & D16S3407 & 0 & FAM & $195-205$ & 0.77 \\
\hline $17 p$ & D17S1866 & 0 & HEX & $144-195$ & 0.80 \\
\hline & D17S926 & 0.6 & FAM & $243-260$ & 0.80 \\
\hline
\end{tabular}

Table 2 (Continued)

\begin{tabular}{|c|c|c|c|c|c|}
\hline Telomere & $\begin{array}{l}\text { D } \\
\text { Number }\end{array}$ & $\begin{array}{l}\text { Genetic } \\
\text { distance } \\
\text { to } \\
\text { telomere } \\
\text { (cM) }\end{array}$ & Fluorophore & Size range & $\begin{array}{l}\text { Hetero- } \\
\text { zygosity }\end{array}$ \\
\hline \multirow[t]{2}{*}{$17 q$} & D17S928 & 0 & NED & $76-96$ & 0.75 \\
\hline & D17S1830 & 11 & HEX & $108-122$ & 0.79 \\
\hline \multirow[t]{2}{*}{$18 p$} & D18S59 & 0.1 & HEX & $127-182$ & 0.81 \\
\hline & D18S476 & 1.6 & HEX & $263-275$ & 0.76 \\
\hline \multirow[t]{2}{*}{$18 q$} & D18S70 & 0 & FAM & $115-135$ & 0.83 \\
\hline & D18S1141 & 1.9 & NED & $263-293$ & 0.77 \\
\hline \multirow[t]{3}{*}{$19 p$} & D19S886 & 0 & NED & $105-165$ & 0.63 \\
\hline & D19S883 & 5.5 & HEX & $158-170$ & 0.72 \\
\hline & D19S878 & 6.5 & FAM & $208-230$ & 0.83 \\
\hline \multirow[t]{2}{*}{$19 q$} & D19S890 & 0 & HEX & $190-210$ & 0.68 \\
\hline & D19S218 & 2.5 & FAM & $240-256$ & 0.60 \\
\hline \multirow[t]{2}{*}{$20 p$} & D20S864 & 0 & FAM & $215-235$ & 0.52 \\
\hline & D20S103 & 0 & FAM & $92-106$ & 0.70 \\
\hline \multirow{2}{*}{$20 q$} & D20S173 & 0 & HEX & $135-195$ & 0.66 \\
\hline & D20S171 & 2.1 & FAM & $123-149$ & 0.73 \\
\hline \multirow[t]{2}{*}{$21 p$} & D21S1911 & 0.1 & HEX & $105-145$ & 0.69 \\
\hline & D21S1904 & 0 & HEX & $152-170$ & 0.52 \\
\hline \multirow[t]{2}{*}{$21 q$} & D21S1897 & 0 & FAM & $178-224$ & 0.74 \\
\hline & D21S1903 & 0.7 & FAM & $243-265$ & 0.83 \\
\hline \multirow[t]{2}{*}{$22 q$} & D22S1169 & 0 & FAM & $110-135$ & 0.78 \\
\hline & $\mathrm{D} 22 \mathrm{~S} 1161$ & 2.2 & HEX & $235-245$ & 0.68 \\
\hline \multirow[t]{2}{*}{$X p$} & DXYS233 & 0 & HEX & $260-300$ & 0.81 \\
\hline & DXYS234 & 2 & NED & $234-254$ & 0.72 \\
\hline \multirow[t]{2}{*}{$X q$} & DXS8087 & 0 & FAM & $270-300$ & 0.69 \\
\hline & DXS1073 & 1.6 & HEX & $217-243$ & 0.80 \\
\hline
\end{tabular}

Genetic distance from centromere and heterozygosity score were obtained from the Genethon map (http://www.genethon.fr).

tions. An internal ROX-labelled size standard (400HD-ROX $1 \mathrm{fmol}$ per lane; PE Biosystems) was added to each lane to ensure precise sizing of PCR products and minimise lane-tolane variation. Gel lane tracking and sizing of the size standard peaks were checked manually for all lanes. GENESCAN $^{\mathrm{TM}}$ and Genotyper ${ }^{\mathrm{TM}}$ softwares (Applied Biosystems) were used to size the PCR products and to analyse the data according to the manufacturer's instructions.

\section{Chromosome and FISH studies}

Metaphase spreads were prepared from phytohemaglutinin (PHA) stimulated blood lymphocyte cultures using standard procedures of hypotonic treatment and methanol/acetic acid fixation (3:1). RHG and GTG banding methods were performed according to standard protocols. ${ }^{14}$

Two sets of subtelomeric FISH probes were used for hybridisation. The first set of probes corresponds to the Chromoprobe Multiprobe T System commercially distributed by Cytocell and is composed of telomeric-specific cosmids and PAC clones previously tested on unrelated individuals to exclude polymorphisms. ${ }^{9}$ The second set of probes is composed of 41 well characterised CEPH YACs specific of each subtelomeric region and located about $2-3 \mathrm{Mb}$ away from the telomere. ${ }^{15}$ They were kindly provided by Thomas Haaf. YAC DNA were isolated by pulse-field gel electrophor- 
Table 3 Sets of PCR-amplified dinucleotide repeat microsatellite markers suitable for automated fluorescent genotyping

\begin{tabular}{|c|c|c|c|}
\hline Locus & Telomere & Labelling & Allele size range \\
\hline \multicolumn{4}{|l|}{ SET 1} \\
\hline D1S243 & $1 p$ & FAM & $142-170$ \\
\hline D1S2682 & $1 q$ & NED & $110-150$ \\
\hline D2S2268 & $2 p$ & HEX & $205-231$ \\
\hline D4S3038 & $4 p$ & NED & $195-240$ \\
\hline D3S3550 & $3 q$ & FAM & $230-270$ \\
\hline \multicolumn{4}{|l|}{ SET 2} \\
\hline D2S2338 & $2 q$ & FAM & $155-173$ \\
\hline D3S1270 & $3 p$ & NED & $154-196$ \\
\hline D5S678 & $5 p$ & NED & $252-264$ \\
\hline D5S2006 & $5 q$ & HEX & $135-165$ \\
\hline D6S281 & $6 q$ & HEX & $203-219$ \\
\hline \multicolumn{4}{|l|}{ SET 3} \\
\hline D4S2930 & $4 q$ & NED & $216-236$ \\
\hline D6S1600 & $6 p$ & FAM & $165-210$ \\
\hline D7S2423 & $7 q$ & HEX & $230-270$ \\
\hline D8S1836 & $8 q$ & HEX & $121-160$ \\
\hline D9S1779 & $9 p$ & NED & $114-154$ \\
\hline \multicolumn{4}{|l|}{ SET 4} \\
\hline D7S2477 & $7 p$ & FAM & $132-180$ \\
\hline D8S504 & $8 p$ & FAM & $193-203$ \\
\hline D9S1838 & $9 q$ & NED & $155-175$ \\
\hline D11S968 & $11 \mathrm{q}$ & HEX & $131-165$ \\
\hline D12S357 & $12 q$ & HEX & $260-315$ \\
\hline \multicolumn{4}{|l|}{ SET 5} \\
\hline D10S249 & $10 p$ & NED & $106-152$ \\
\hline D10S1700 & $10 q$ & FAM & $107-148$ \\
\hline D11S1363 & $11 p$ & FAM & $240-260$ \\
\hline D12S352 & $12 p$ & HEX & $145-170$ \\
\hline \multicolumn{4}{|l|}{ SET6 } \\
\hline D14S1007 & $14 q$ & HEX & $110-135$ \\
\hline D15S966 & $15 q$ & HEX & $225-265$ \\
\hline D16S521 & $16 p$ & FAM & $156-172$ \\
\hline D16S3407 & $16 q$ & FAM & $195-205$ \\
\hline D13S293 & $13 q$ & NED & $80-106$ \\
\hline \multicolumn{4}{|l|}{ SET 7} \\
\hline D17S1866 & $17 p$ & FAM & $144-195$ \\
\hline D17S928 & $17 q$ & NED & $76-96$ \\
\hline D18S59 & $18 p$ & HEX & $127-182$ \\
\hline D18S70 & $18 q$ & FAM & $115-135$ \\
\hline D19S886 & $19 p$ & NED & $105-165$ \\
\hline \multicolumn{4}{|l|}{ SET 8} \\
\hline D19S890 & $19 q$ & HEX & $190-210$ \\
\hline D20S864 & $20 p$ & FAM & $215-235$ \\
\hline D21S1911 & $21 p$ & HEX & $105-145$ \\
\hline D22S1169 & $22 q$ & FAM & $67-85$ \\
\hline DXYS233 & $X p$ & HEX & $260-300$ \\
\hline \multicolumn{4}{|l|}{ SET 9} \\
\hline D20S173 & $20 q$ & HEX & $135-195$ \\
\hline D21S1897 & $21 q$ & FAM & $178-224$ \\
\hline DXS8087 & $\mathrm{Xq}$ & FAM & $270-300$ \\
\hline
\end{tabular}

esis (PFGE) and amplified using the degenerate oligonucleotide-primed-polymerase chain reaction (DOP-PCR) proce- dure as previously described. ${ }^{16}$ Probes were labelled with biotin-16-dUTP or digoxigenin-11-dUTP (Boehringer-Mannheim) using a commercially available random-priming kit (Gibco-BRL). Biotin-labelled probes were detected using Texas Red (TR) conjugated to avidin and digoxigenin-labelled probes were detected using fluorescein isothiocyanate (FITC) conjugated to anti-digoxigenin. Slides were counterstained with 4', 6'-diamidino-2-phenylindole (DAPI). Image capture and analyses were performed using a Zeiss Axiophot epifluorescence microscope equipped with the appropriate filter combination for detecting TR, FITC and DAPI. The images were captured by a cooled CCD camera controlled using an image analysis system (Vysis). Ten hybridised metaphases were analysed for each probe.

\section{Results}

Analysis of chromosome ends using automated fluorescent genotyping

We first designed a set of 42 fluorescently labelled primer pairs that corresponded to the two most distal microsatellite markers ( 2 bp repeat) of the Genethon human linkage map. ${ }^{17}$ Each pair was labelled with one of the three fluorescent dyes FAM, HEX or NED. For each individual, DNA was individually amplified for all loci. PCR products were then arranged into nine sets on the bases of allele sizes and colours of fluorescent labelling (Table 3) and electrophoresed in one lane on an automated sequencer (Figure 1A). Sizing of the fragments was then performed using the ABI GeneScan software (Figure 1B). For each marker, the genotype of the child was determined and compared to the parental genotypes to detect (i) missing alleles (deletion), (ii) the presence of a third allele (duplication) or (iii) the presence of one or two alleles from one parent with no contribution from the other parent (uniparental isodisomy or heterodisomy respectively). Whenever the child was heterozygous for two different alleles identical to that of his parents, the genotype was regarded as normal. In case of uninformative polymorphism, when the child was homozygous for one allele shared by both parents, the segregation of the closest microsatellite marker on the Genethon map was tested. A total of 29 children, belonging to 24 different families, and 48 parents were analysed and a total of 4478 genotypes were determined. We detected six cases of non-mendelian inheritance.

Identification of a $\mathbf{t}(6,10)$ translocation in family 1

The two affected children of family 1 presented abnormal genotypes for both chromosome $6 \mathrm{q}$ and $10 \mathrm{q}$ markers. The son inherited a single maternal allele at the D6S281 locus, suggesting therefore a telomeric deletion of chromosome $6 \mathrm{q}$ (Figure 2A). His sister had a normal genotype at this locus but inherited a single maternal allele at the D10\$1700 locus (Table 4). To confirm these findings and estimate the extent of the imbalance, additional genotyping was performed with markers selected from the CEPH-GENETHON integraded 

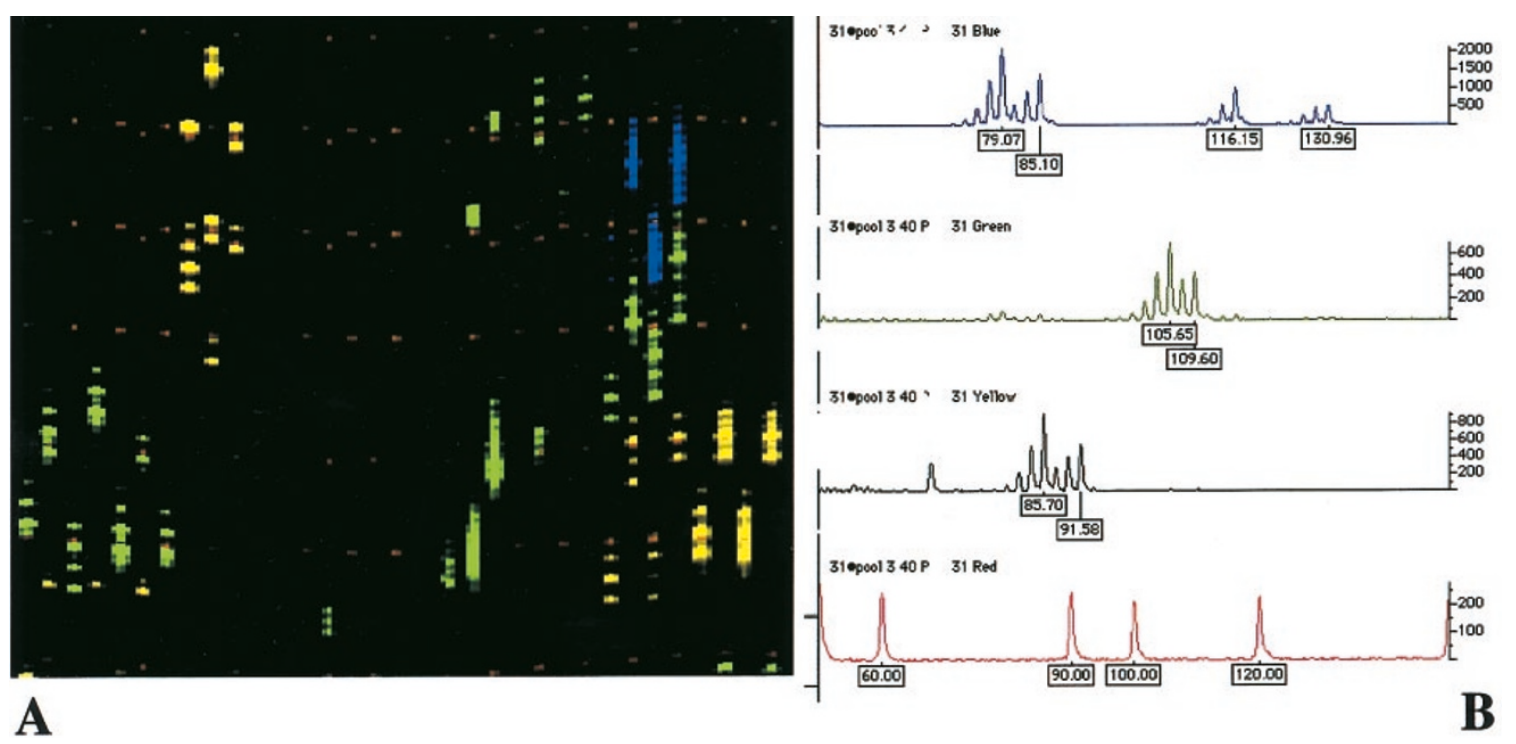

A

Figure 1 Extensive screening of children with idiopathic mental retardation. A Gel view from the ABI PRISM 377 DNA sequencer showing samples from 36 individuals (one per lane) and the ladder. The four loci are multiplexed on the bases of allele size and colours of fluorescent labels. The red bands are the internal size standards. B Genotyper electropherogram plots for one individual. The peaks shown in blue, green and black represent the amplified PCR products labelled with FAM, HEX and NED respectively.
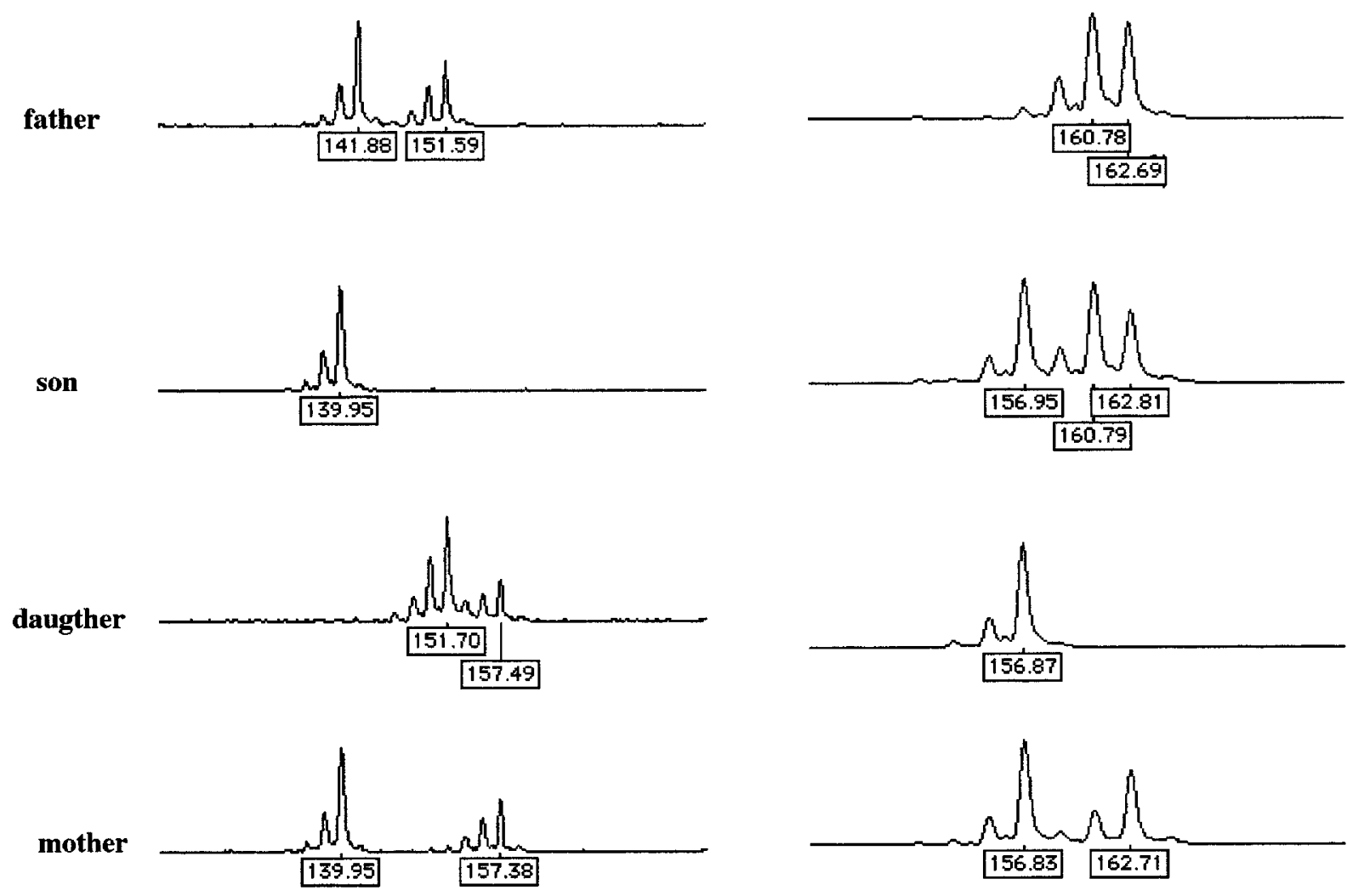

A

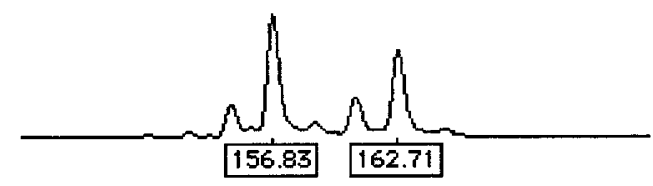

B

Figure 2 Identification of a telomeric rearrangement in family 1. Genotyping data of the four members of family 1 typed with D6S281, A and D10S1676, B. The results show that the son failed to inherit the paternal chromosome 6 allele and is trisomic for the chromosome 10 marker. His sister is missing the paternal allele for the chromosome 10 markers. 
Table 4 Genotype analysis with chromosome 6, 10, 16 and 17 markers

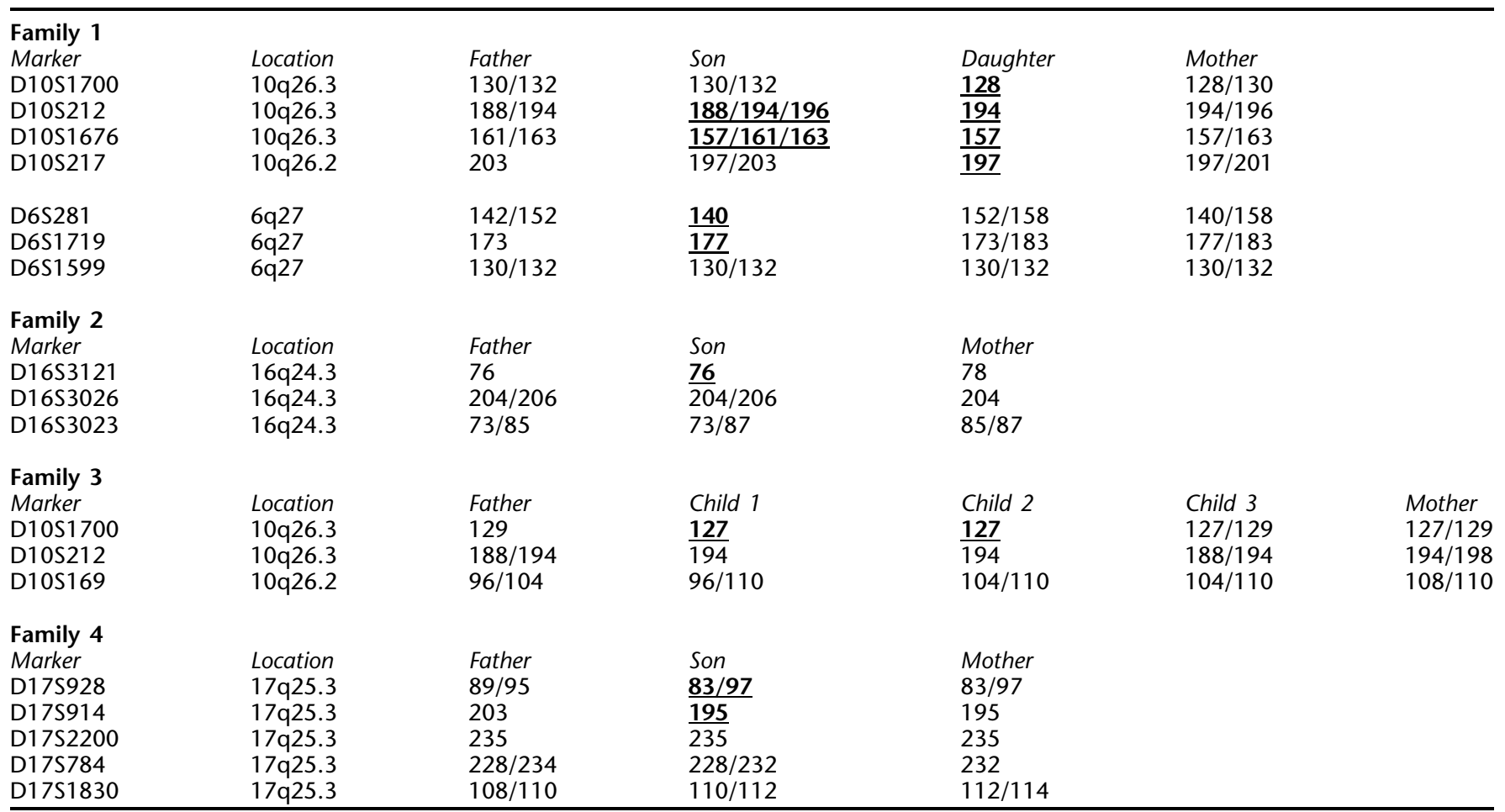

Markers used are shown on the left and size of the different alleles are given in base pairs for the parents and the affected child. Genotypes informative for monosomy/trisomy or maternal disomy are indicated in bold underlined characters.

map database. Our data clearly demonstrate that the two children have inherited opposite derivatives of a balanced paternal translocation $\mathrm{t}(6 ; 10)(\mathrm{q} 27 ; \mathrm{q} 26)$, resulting in a monosomy 6q27/trisomy $10 q 26$ in the son and in a monosomy 10q27 in the daughter (Figure 2B and Table 4). The rearrangement extends up to the D6S1719 locus on chromosome 6 and up to the D10S217 locus on chromosome 10 (Table 4). Based on the marker position, we estimated that the rearrangement involves a region of at least $15 \mathrm{cM}$ on chromosome 6 and $18 \mathrm{cM}$ on chromosome 10. FISH analysis confirmed the balanced rearrangement in the father and demonstrated that the daughter is trisomic for the region 6q27 (Figure 3). Since the genotype of the daughter at the locus D6S281 is normal, a crossing over between the translocated part of the chromosome 6 and the normal chromosome 6 must have occurred (Figure 4). The crossing over should have occurred during meiosis I in the carrier father so that translocated part of the chromosome 6 on the der(10) was exchanged with the corresponding part of the intact chromosome 6 copy.

Chromosome $16 \mathrm{q}$ anomaly in family 2

In family 2, a maternal non contribution was detected at the D16S3121 locus on chromosome 16q while flanking markers were regularly inherited (Table 4). FISH analysis using chromosome $16 \mathrm{q}$ probes detected two normal signals in all metaphase spreads examined (data not shown). Since nonpaternity was clearly excluded, this result could be accounted for by either an allele mutation in the child (so that the final repeat length is the same as length of the father allele) or a small deletion undetectable using the Cytocell probes.

\section{Monosomy 10qter in family 3}

In family 3 , two severely affected children (cases 1 and 2) inherited a single maternal allele and no paternal allele at the D10S1700 locus on chromosome 10q while a mildly affected child (case 3) inherited one allele from each parent (Table 4). Genotyping the rearrangement with additional markers revealed that the breakpoint maps distal to D10S169 so that the deleted region is less then $3 \mathrm{cM}$ in size. FISH analysis of this family was unremarkable, a feature which should be related to the proximal location of the FISH probes with respect to the breakpoint. In the absence of any evidence for segmental trisomy in the third child, and because of the results of FISH analyses, we are presently unable to discriminate between a germinal mosaicism for a paternal deletion or the unbalanced inheritance of a balanced paternal translocation.

\section{Maternal heterodisomy in family 4}

The proband of family 4 has failed to inherit a paternal allele at the D17S928 locus but carried a maternal heterodisomy for 


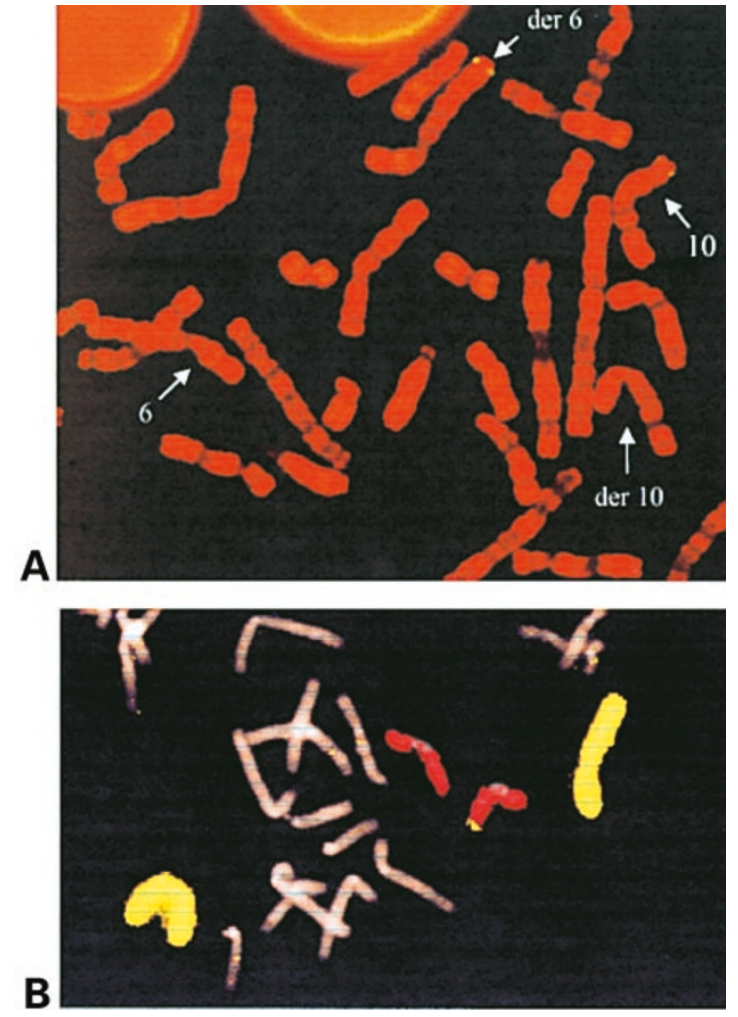

Figure 3 FISH studies in family 1. (A) FISH analysis was performed with a PAC probe containing marker D10S217 and revealed a balanced translocation in the carrier father. Two signals were present which correspond to the normal chromosome 10 homologue or to the derivative chromosome 6 . The two other unlabelled normal chromosome 6 and derivative chromosome 10 are also indicated. (B) Whole chromosome painting using chromosomes 6 and 10 probes on chromosome of the daughter confirms the presence of an unbalanced karyotype.

chromosome $17 \mathrm{q}$ markers (Table 4 ). Genotyping of additional markers confirmed the absence of paternal contribution for this region and indicated that the extent of the maternal disomy was smaller than $11 \mathrm{cM}$. Analysis of the propositus and his parents with STSs from other chromosomes showed typical mendelian inheritance. A detailed analysis of this case will be described elsewhere (Rio et al, personal communication). Since uniparental disomies (UPD) are frequently associated with confined placental mosaicism, we cannot conclude whether the abnormal phenotype results from the dosage of an imprinted gene or from mosaicism for trisomy $17 \mathrm{q}$.

\section{Discussion}

Here, we report on a novel and efficient automated strategy based on the fluorescent genotyping technique to screen for cryptic telomeric rearrangements in children with develop-

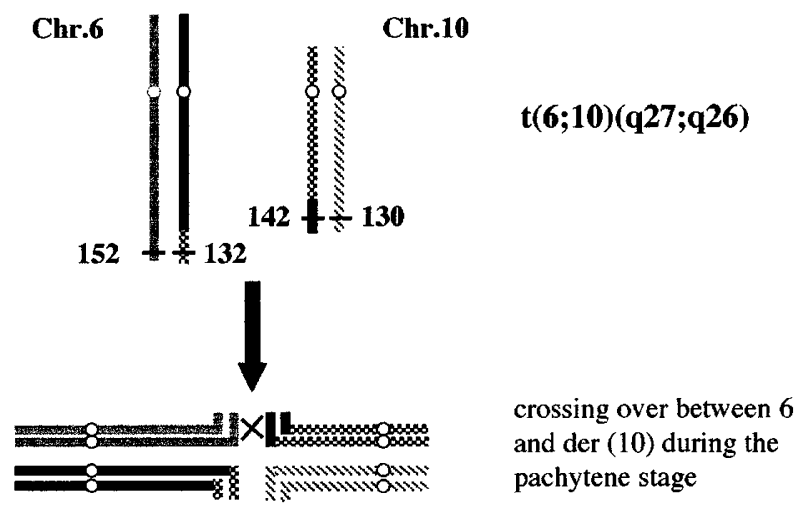

M1 adjacent 1 segregation

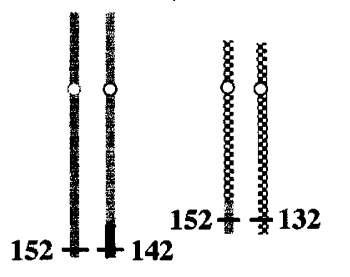

M2

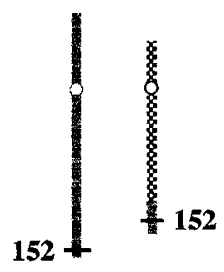

Isodisomy 6qter

Nullisomy 10qter

Figure 4 Proposed mechanism of the recombination event leading to the genotype of the daughter in family 1 . A crossing over must have occurred during the pachytene stage, when the two translocated chromosomes and their normal counterparts come together as a quadrivalent. After adjacent 1 segregation and the second division, this recombination leads to isodisomic 6 ter/nullisomic 10qter gamete. The genotypes for the D10S1700 and D6S281 loci are indicated.

mental delay and multiple congenital anomalies. Despite normal conventional karyotypes in the 29 probands, we identified three chromosomal abnormalities in our series (about 10\%). The prevalence of subtle telomeric abnormalities was first estimated to $7.4 \%$ using cytogenetic detection methods. ${ }^{10}$ A subsequent study based on the screening for submicroscopic rearrangements using microsatellite markers suggested a higher frequency (18\%) and concluded that genotyping might be more sensitive than previously used 
FISH techniques. ${ }^{13}$ Our results give additional support to this conclusion. Moreover, in contrast to cytogenetics (which requires a high technical expertise), genotyping offers the advantage of giving quantitative and objective results that can be automated in many respects. Lastly, genotyping can directly identify the parental origin of the rearrangement.

The set of markers described here have an average heterozygosity score of 0.75 . Since only few chromosome maps encompass telomeres, several loci tested (Table 2) are likely to lie hundreds of kilobases away from the telomere. Sequence data derived from the human genome sequencing projects will hopefully allow us to define more informative and/or telomeric microsatellite markers, so that the primary sets of markers described here will be improved. The development of new fluorophores will also allow reduction of the number of sets. We estimated extensive genotyping of one child and his two parents to cost 80 dollars, ie three to four times less than the cost estimated for the cytogenetic method, ${ }^{10}$ and progress in automation is likely to make this approach even more affordable.

However, it should be noted that this approach also has its limitations. First, it cannot detect balanced rearrangements when the dosage is unaltered. A second limitation is that dosage differences cannot be reliably detected. Hence, this technique may overlook cases of monosomy and trisomy when the parents share the same allele or cases of small tandem duplications. Finally, this screening technique may generate false-positive results due to the instability of microsatellites, as probably observed in family 2. Thus, non mendelian inheritance of a single microsatellite by itself does not provide evidence that a chromosomal rearrangement has occurred. Genotyping of additional markers and/or FISH analyses are required to support this conclusion. In addition, FISH analyses are required to distinguish a de novo rearrangement from an inherited chromosome imbalance and to identify the carrier(s) of the balanced anomaly in the family.

Finally, the microsatellite technique provides the unique opportunity to detect uniparental disomies, a major disease causing mechanism. ${ }^{18}$ This feature is illustrated by the results of family 4 where the proband carries a maternal heterodisomy of the telomere of chromosome 17q. On the basis of the haplotype analysis, the mechanism responsible for this partial UPD of chromosome 17 is likely to be the consequence of the fertilization of a maternal disomic gamete (due to a nondisjunction error) by a monosomic paternal gamete. Postzygotically, a recombination must have occurred between one of the maternal chromosome 17 and the paternal one. Subsequently, and independently, the maternal chromosome 17 has been lost in the progenitor cell line. In this family, the abnormal phenotype could result from either confined placental mosaicism or the imprinting of this part of the genome. Placental tissues were unavailable in this case so we could not test for trisomy 17 in these cells. However, confined placental mosaicism is usually associated with intrauterine/post-natal growth retardation, a feature which was not observed in the proband. On the other hand, maternal isodisomy for chromosome 17 has previously been described in a 2-year-old boy with normal growth and psychomotor development, ${ }^{19}$ and the terminal long arm of chromosome 17 is not known to undergo imprinting. ${ }^{20}$ Further investigations are therefore required to know whether this region contains one or several imprinted gene(s).

Finally, in addition to its clinical relevance, this novel strategy will hopefully allow the delineation of new contiguous gene syndromes and the identification of new imprinted regions.

\section{Acknowledgements}

We would like to acknowledge the families involved in this study for providing samples. We also thank Suzie Lefebvre for critical reading of the manuscript. This work was supported by Centre National de la Recherche Scientifique and by Fondation pour la Recherche Médicale.

\section{References}

1 Gustavson KH, Hagberg B, Hagberg G, Sars K: Severe mental retardation in a Swedish county: II, etiologic and pathogenetic aspects of children born 1959-1970. Neuropediatry 1977; 8: $293-304$.

2 Elwood JH, Darragh PM: Severe mental handicap in Northern Ireland. J Mental Defic Res 1973; 25: $147-155$

3 Flint J, Wilkie AOM: The genetics of mental retardation. Br Med Bull 1996; 52: $435-464$.

4 Lamb J, Wilkie AOM, Harris PC et al: Detection of breakpoints in submicroscopic chromosomal translocation, illustrating an important mechanism for genetic disease. Lancet 1989; ii; $819-824$.

5 Wilkie AOM, Buckle VJ, Harris PC et al: Clinical features and molecular analysis of the alpha thalassemia/mental retardation syndromes. I. Cases due to deletions involving chromosome band 16p13.3. Am J Hum Genet 1990; 46: 1112-1126.

6 Goodship J, Curtis A, Cross I et al: A submicroscopic translocation, $\mathrm{t}(4 ; 10)$, responsible for recurrent Wolf-Hirschhorn syndrome identified by allele loss and fluorescent in situ hybridization. J Med Genet 1992; 29: 451-454.

7 Kuwano A, Ledbetter SA, Dobyns WB, Emanuel BS, Ledbetter $\mathrm{DH}$ : Detection of deletions and cryptic translocations in MillerDiecker syndrome by in situ hybridization. Am J Hum Genet 1991; 49: $707-714$.

8 Overhauser J, Bengtsson U, McMahon J et al: Prenatal diagnosis and carrier detection of a cryptic translocation by using DNA markers from the short arm of chromosome 5. Am J Hum Genet 1989; 45: 296-303.

9 Knight SJL, Horsley SW, Regan R et al: Development and clinical application of an innovative fluorescence in situ hybridization technique which detects submicroscopic rearrangements involving telomeres. Eur J Hum Genet 1997; 5: 1-8.

10 Knight SJL, Regan R, Nicod A et al: Subtle chromosomal rearrangements in children with unexplained mental retardation. Lancet 1999; 54: 1676-1681.

11 Ghaffari SR, Boyd E, Tolmie JL, Crow YJ, Trainer AH, Connor JM: A new strategy for cryptic telomeric translocation screening in patients with idiopathic mental retardation. J Med Genet 1998; 35: $225-233$. 
12 Rosenberg MJ, Vaske D, Killoran CE et al: Detection of chromosomal aberrations by a whole-genome microsatellite screen. Am J Hum Genet 2000; 66: 419-427.

13 Slavotinek A, Rosenberg M, Knight S et al: Screening for submicroscopic chromosome rearrangements in children with idiopathic mental retardation using microsatellite markers for the chromosome telomeres. J Med Genet 1999; 36: 405-411.

14 de Grouchy J, Turleau C: Clinical Atlas of human chromosome. John Wiley and sons, New York; 2nd ed, 1984.

15 Kingsley K, Wirth J, van der Maarel S, Freier S, Ropers HH, Haaf $\mathrm{T}$ : Complex FISH probes for the subtelomeric regions of all human chromosomes: comparative hybridization of $\mathrm{CEPH}$ YACs to chromosomes of the Old World monkey Presbytis cristata and great apes. Cytogenet Cell Genet 1997; 78: 12-19.

16 Telenius H, Carter NP, Bebb CE, Nordenskjold M, Ponder BA, Tunnacliffe A: Degenerate oligonucleotide-primed PCR: general amplification of target DNA by a single degenerate primer. Genomics 1992; 13: 718-725.
17 Colette D, Faure S, Fizames C et al: A comprehensive genetic map of the human genome based on 5,264 microsatellites. Nature 1996; 380: 152-154.

18 Morison IE, Reeve AE: A catalogue of imprinted gene and parent-of-origin effects in humans and animals. Hum Mol Genet 1998; 7: $1599-1609$.

19 Genuardi M, Tozzi C, Pomponi MG et al: Mosaic trisomy 17 in aminocytes: phenotypic outcome, tissue distribution, and uniparental disomy studies. Eur J Hum Genet 1999; 7: 421 - 426.

20 Kotzot D: Abnormal phenotypes in uniparental disomy (UPD): fundamental aspects and a critical review with bibliography of UPD other than 15. Am J Hum Genet 1999; 82: 265-274. 